\section{Effect of Exercise Training on Heart Rate Variability in Patients with Heart Failure After Percutaneous Coronary Intervention}

\author{
Abolahrari-Shirazi S. ${ }^{1}$, Kojuri J. ${ }^{2}$, Bagheri Z. ${ }^{3}$, Rojhani-Shirazi \\ Z. $4,5 *$
}

\begin{abstract}
Background: This study aims to evaluate the effect of exercise training on heart rate variability (HRV) and to determine the correlation between parameters of HRV and the ejection fraction in patients with heart failure after percutaneous coronary intervention.

Material and Methods: Fifty patients with left ventricular ejection fraction $\leq 40 \%$ undergone percutaneous coronary intervention were randomly allocated in either an exercise training (ET) group or a control group. The ET group performed exercise training for 45 minutes, three times a week for seven weeks. Patients in both groups received a leaflet for daily exercising at home. HRV parameters comprising, the standard deviation of normal R-R intervals (SDNN), the square root of the mean of the squares of successive R-R intervals differences (RMSSD), the percentage of successive R-R intervals differing from more than $50 \mathrm{~ms}$ (PNN50), using 24-hour Holter electrocardiographic monitoring was measured.
\end{abstract}

Results: After the intervention, the SDNN improved in the ET group $(\mathrm{P}=0.002)$, while changes in all remaining HRV indices were insignificant $(P \geq 0.05)$. The control group showed no significant changes in any HRV parameters $(\mathrm{P} \geq 0.05)$. Changes in SDNN in the ET group were significantly different from the control group $(\mathrm{P}=0.003)$. At baseline, our results revealed a significant weak correlation between ejection fraction and SDNN ( $\mathrm{r}=0.279, \mathrm{P}=0.047)$. However, ejection fraction did not correlate significantly with RMSSD and PNN50.

Conclusion: Exercise training is safe and feasible in post percutaneous coronary intervention patients, even in those with reduced ejection fraction. In a seven-week period, exercise training was effective in improving HRV in heart failure patients after percutaneous coronary intervention.

\section{Keywords}

Exercise Training, Heart Failure, Percutaneous Coronary Intervention, Heart Rate Variability

\section{Introduction}

\footnotetext{
oronary artery disease is a major reason for the heart failure [1]. Although percutaneous coronary intervention (PCI) is an effective approach to restore myocardial perfusion, in some patients, heart failure develops even after PCI $[2,3]$. Left ventricular dysfunction is associated with an increased risk of sudden cardiac death [1]. Optimal medical therapy and cardiac rehabilitation in conjunction with PCI can be beneficial in diminishing the impact of heart failure [3].
}

${ }^{1}$ PhD Candidate, Physical Therapy Department, School of Rehabilitation Sciences, Shiraz Univer-

sity of Medical Sciences, Shiraz, Iran

${ }^{2}$ Cardiologist, Inter-

ventionist, Full profes-

sor, Clinical education

improvement research

center, Shiraz University

of Medical Sciences,

Shiraz, Iran

${ }^{3} \mathrm{PhD}$ of Biostatistics,

Department of Biosta-

tistics, Shiraz University

of Medical Sciences,

Shiraz, Iran

${ }^{4} \mathrm{PhD}$ of Physiotherapy,

Associated professor,

Physical Therapy Depart

ment, School of Rehabil-

itation Sciences, Shiraz

University of Medical

Sciences, Shiraz, Iran

${ }^{5}$ Rehabilitation Sciences

Research Center, Shiraz

University of Medical sci-

ences, Shiraz, Iran

*Corresponding author:

Z. Rojhani-Shirazi

School of Rehabilitation

Sciences, Shiraz Univer-

sity of Medical Sciences,

Chamran Blvd., Abiverdi

1Street, P.O.Box: 71345

1733, Shiraz, Iran

E-mail: rojhaniz@sums. ac.ir

Received: 26 September 2017 Accepted: 14 October 2017 
Exercise training is well-established nonpharmacological therapy for heart failure patients [4]. Several studies have shown that the exercise can be an asset for heart failure patients, improving their functional capacity [5], quality of life [6,7], cardiac [8] and vascular functions [9], and reducing mortality and morbidity [10].

Heart rate variability is a physiological feature that reflects the impact of the autonomic nervous system on the cardiac function. Depressed heart rate variability (HRV) has been confirmed to be a prognostic marker and is correlated with morbidity and mortality [1113]. However, HRV is not utilized in the current clinical practice. Establishing the correlation between HRV parameters and an ejection fraction can help verify the validity of HRV as a clinical tool for evaluating the response to therapeutic interventions in patients with heart failure. Also exercise training has been reported in order to improve HRV in patients following PCI $[14,15]$; however, no study has focus specifically on the impact of exercise therapy on HRV in heart failure patients after PCI.

The aim of the present study is to investigate the effect of exercise training on HRV indices and to examine the correlation between these parameters and the ejection fraction in patients with heart failure after the percutaneous coronary interventions.

\section{Materials and Methods}

\section{Study Design and Participants}

This randomized, controlled, parallel-group trial investigated the effect of exercise training on heart rate variability in patients with heart failure after percutaneous coronary intervention.

All patients undergone percutaneous coronary intervention and referred to the Cardiac Rehabilitation Department of Al-Zahra Heart Charity Hospital in Shiraz from October 2015 to November 2016 by their cardiologist, one month after angioplasty, were evaluated for inclusion in this study. The inclusion criteria were age 40-70 years, left ventricular ejection fraction $\leq 40 \%$, and New York Heart Association functional class I, II or III. Patients were excluded from the study if the following criteria were met: severe exercise intolerance, uncontrolled arrhythmia, uncontrolled angina pectoris, severe kidney or lung diseases, musculoskeletal or neuromuscular conditions preventing exercise testing or training, and signs or symptoms of ischemia $[16,17]$. All participants signed an informed consent form before participating in the study. Ethics approval was obtained from the Shiraz University of Medical Sciences Ethics Committee (reference number: IR.SUMS.REC.1394113).

\section{Randomization and Masking}

After the baseline assessment and before the start of treatment protocols, the 50 patients were randomly allocated in either an exercise training (ET) group or a control group. A biostatistician generated the random allocation sequence using the permuted block randomization method. The random allocation sequence was implemented by a nurse who was unaware of the study hypothesis and intervention. All participants, referring physicians, research assistants and outcome assessors were blinded to a group assignment. All data were gathered independently by personnel blinded to the study aims, and analyzed by an analyst who was unaware of treatment allocation.

\section{Measurements}

\section{Exercise Testing}

In order to predict peak $\mathrm{VO}_{2}$, a supervised graded exercise test to record volitional fatigue or symptoms was conducted on a treadmill using Bruce protocol at baseline. The test was done in nonfasting conditions and under medication. A 12-lead ECG was recorded continuously, and blood pressure was measured with an arm cuff every 3 min.

Heart Rate Variability

Heart rate variability was recorded with a 
24-hour Holter electrocardiographic monitor with three leads at a sampling rate of $250 \mathrm{~Hz}$ (Click Holter, Cardioline, Milan, Italy) before and after the seven-week intervention period. HRV parameters were obtained from Cardioline Holter software. The ECG recordings were examined by a specialist who was blinded to the participant group allocation. All ectopic beats, noisy data and artefacts were filtered out prior to calculating HRV parameters, and only the normal R-R intervals over a period of at least 15 hours of signal were analyzed. The time domain parameters considered in this study were the standard deviation of normal R-R intervals (SDNN), the square root of the mean of the squares of successive R-R intervals differences (RMSSD), the percentage of successive R-R intervals differing by more than $50 \mathrm{~ms}$ (PNN50).

\section{Interventions}

The patients were randomly allocated to one of two groups: ET or control. All patients in exercise group received training three times a week for seven weeks. Training sessions were supervised by an experienced physiotherapist and a nurse. Electrocardiogram and heart rate were monitored with telemetric monitoring, and blood pressure was measured at the beginning and at the end of each session. Each training session started with warm-up for $5 \mathrm{~min}$ utes and ended with 5 minutes of cool-down consisting of light aerobic exercise. Patients in both groups received a leaflet for daily exercising at home. Briefly, the exercises included ten types of active exercises in trunk, upper and lower limbs. The patient was asked to do each exercise for 10 repetitions. Each exercise session at home lasted 15-20 minutes.

\section{ET Protocol}

The patients performed exercise training for a total of 45 minutes at $40 \%$ to $70 \%$ peak $\mathrm{VO}_{2}$ predicted with a supervised graded exercise test on a treadmill with the Bruce protocol. Exercise training consisted of exercise on a cycle ergometer for 20 minutes, an arm er- gometer for 10 minutes and a treadmill for 15 minutes. At the beginning, training intensity was set at $40 \%$ peak $\mathrm{VO}_{2}$, and then increased progressively to $70 \%$ peak VO2 $[18,19]$. As training intensity increased, the patients' heart rate, rate of perceived exertion (Borg scale) and cardiac symptoms were also considered.

Control Protocol

Patients in the control group received a leaflet for daily exercising at home as explained above.

\section{Statistical Analysis}

According to the previous studies, the sample size was determined to be 25 patients in each group, when $\alpha=0.05$ and $\beta=0.2$ by the NCSS (PASS) 2007 software. All analyses were done with SPSS software, v. 16 on an intention-totreat principle. Normality of the data distribution was checked with the Shapiro-Wilk test. We used descriptive statistics including mean \pm SD for quantitative variables and the frequency (percentage) for qualitative variables to summarize the participants' baseline characteristics. Chi square test was used to compare qualitative variables among our three groups. We used the Spearman's bivariate correlation test to determine the correlation between the ejection fraction and HRV parameters. Paired t-tests and Wilcoxon signed rank tests were used for within-group comparisons for variables with normal and abnormal (PNN50) distribution, respectively. For each participant, the change in values from baseline was calculated at seven weeks (post intervention). Between-group comparisons were tested with independent t-test and the Mann-Whitney test, and $\mathrm{P}<0.05$ was considered significant for all tests.

\section{Results}

We screened 205 patients, 152 of whom were excluded because they did not meet the eligibility criteria, and three patients declined participation. Fifty patients were randomized to each of the two groups. Three participants 
withdrew from the study because of loss of interest and severe knee pain. Participants completed the study without any serious adverse events. Premature ventricular contractions were recorded in two patients. One patient in ET group experienced severe hypotension during two consecutive training sessions which were normalized after exercise discontinuation. Another patient in ET group had the respiratory problem that was not related to exercise training and had to be hospitalized for three days. These last two patients resumed training after one week.

Our results showed no statistically significant differences between groups at baseline for demographic and clinical characteristics (Table 1). There were also no significant differences between groups for heart rate variability parameters at baseline $(\mathrm{P} \geq 0.05)$.

Changes in parameters after the intervention in both groups are presented in Table 2. After seven weeks of exercise training, SDNN increased after the intervention in the ET group $(\mathrm{P}=0.002)$, while changes in RMSSD and PNN50 were not significant in training group $(\mathrm{P} \geq 0.05)$. The control group showed no significant changes in any heart rate variability parameter $(P \geq 0.05)$. The results of independent t-test revealed statistically significant differences between the two groups with respect to the mean change values in $\operatorname{SDNN}(\mathrm{P}=0.003)$. There were no significant differences amongst groups regarding changes in remaining outcomes ( $\mathrm{P} \geq 0.05$, Table 3 ).

Our results demonstrated a significant correlation between the ejection fraction and SDNN at baseline $(r=0.279, \mathrm{P}=0.047)$. However, ejection fraction did not correlate significantly with RMSSD and PNN50 ( $\mathrm{P}=0.246$ and $\mathrm{P}=0.505$, respectively).

\section{Discussion}

To the best of our knowledge, the present study is the first to investigate the effect of exercise training on HRV in heart failure patients after percutaneous coronary intervention. All
Table 1: Baseline demographic and clinical characteristics of participants.

\begin{tabular}{lccc} 
& $\begin{array}{c}\text { exercise } \\
\text { training } \\
\text { (n=25) }\end{array}$ & $\begin{array}{c}\text { Control } \\
\text { (n=25) }\end{array}$ & $\begin{array}{c}\text { P- } \\
\text { value }\end{array}$ \\
\hline Age(year) & $57.64(7.85)$ & $57.32(9.41)$ & 0.897 \\
\hline Weight(Kg) & $72.64(10.21)$ & $70.28(14.60)$ & 0.511 \\
\hline Height(Cm) & $164.72(7.55)$ & $163.68(11.16)$ & 0.702 \\
\hline BMI(Kg/m $)$ & $26.71(2.91)$ & $26.10(3.86)$ & 0.530 \\
\hline Male/female & $18 / 7$ & $16 / 9$ & 0.544 \\
\hline NYHA I/II/II & $9 / 11 / 5$ & $11 / 10 / 4$ & 0.870 \\
\hline LVEF (\%) & $33.80(6.17)$ & $35.00(5.59)$ & 0.475 \\
\hline Drug therapy & & & \\
\hline ACE-I (\%) & 32 & 32 & 1.000 \\
\hline Aspirin (\%) & 100 & 100 & $\mathrm{NA}$ \\
\hline Plavix (\%) & 100 & 100 & $\mathrm{NA}$ \\
\hline ARBs (\%) & 36 & 32 & 0.765 \\
\hline CCBs (\%) & 12 & 8 & 1.000 \\
\hline Diuretics (\%) & 48 & 40 & 0.569 \\
\hline Statins (\%) & 88 & 92 & 1.000 \\
\hline Nitrates (\%) & 88 & 84 & 1.000 \\
\hline Digoxin (\%) & 16 & 0 & 0.110 \\
\hline Comorbidity & & & \\
\hline Diabetes (\%) & 16 & 20 & 1.000 \\
\hline Hypertension (\%) & 36 & 20 & 0.345 \\
\hline & & & \\
\hline & & & \\
\hline
\end{tabular}

Data are presented as the mean (SD) or number of patients or percentages. NYHA, New York Heart Association; LVEF, left ventricular ejection fraction; ACE-I, angiotensin converting enzyme inhibitors; NA, not applicable; ARB, angiotensin receptor blocker; CCB, calcium channel blocker.

participants completed the study without any serious adverse events, and they admitted that exercise protocols were safe and well tolerated. The main finding of our study was that exercise training was effective only in increasing SDNN in training group. No significant changes in HRV parameters were seen in the control group. A significant difference was seen only for SDNN between the ET and control groups.

Several studies reported improvements in HRV indices in the time domain after exercise training [14, 20-22]. Our results were similar 
Table 2: Within-group comparisons for parameters of heart rate variability.

\begin{tabular}{lcccccc} 
& \multicolumn{3}{c}{ Exercise training $(\mathbf{n = 2 5})$} & \multicolumn{3}{c}{ Control $(\mathbf{n = 2 5 )}$} \\
\cline { 2 - 7 } & Pre & Post & P value & Pre & post & P value \\
\hline SDNN(ms) & $99.27(33.04)$ & $130.96(57.76)$ & 0.002 & $117.67(40.59)$ & $117.69(40.71)$ & 0.998 \\
\hline RMSSD(ms) & $31.45(13.19)$ & $36.26(18.97)$ & 0.079 & $32.75(11.76)$ & $30.67(14.11)$ & 0.460 \\
\hline PNN50 (\%) & $6.90(7.40)$ & $6.42(7.84)$ & 0.986 & $8.22(7.04)$ & $7.30(6.23)$ & 0.715
\end{tabular}

Data are presented as the mean (SD). SDNN, standard deviation of normal R-R intervals; RMSSD, square root of the mean of the squares of successive R-R intervals differences; PNN50, percentages of differences between adjacent $\mathrm{R}-\mathrm{R}$ intervals that are greater than 50 millisecond.

Table 3: Between-group comparisons for changes in parameters of heart rate variability.

\begin{tabular}{lccc} 
& $\begin{array}{c}\text { Exercise } \\
\text { training } \\
(\mathbf{n}=\mathbf{2 5})\end{array}$ & $\begin{array}{c}\text { Control } \\
(\mathbf{n}=\mathbf{2 5})\end{array}$ & $\mathbf{P}$ value \\
\hline SDNN(ms) & $31.68(44.43)$ & $0.01(25.49)$ & 0.003 \\
\hline RMSSD(ms) & $4.81(13.13)$ & $-2.08(13.86)$ & 0.077 \\
\hline PNN50 (\%) & $-0.48(6.05)$ & $-0.91(6.24)$ & 0.992
\end{tabular}

Data are presented as the mean (SD). SDNN, standard deviation of normal R-R intervals; RMSSD, square root of the mean of the squares of successive R-R intervals differences; PNN50, percentages of differences between adjacent $R-R$ intervals that are greater than 50 millisecond.

to those reported by Larsen et al. [21], who demonstrated a significant increase only in the SDNN index, whereas the changes in RMSSD and PNN50 were not statistically significant. Another publication demonstrated that a highintensity interval exercise program was effective in increasing the SDNN and RMSSD indices in patients following PCI for angina pectoris [14]. One hypothesis for the contrasting results of clinical trials to study the effects of exercise in patients with the cardiovascular disease is the different types and intensities of training. In addition, the difference between the population characteristics might account for the dissimilarity between our results and those reported in the mentioned study.
The RMSSD and PNN50 indices reflect vagal modulation, whereas SDNN is an index of total HRV modulated by low-frequency cyclical alterations. These low frequency changes reflect thermoregulatory and peripheral chemoregulatory mechanisms, and variations in activity of the renin-angiotensin system. Therefore, the depressed SDNN in heart failure patients mirrors the summed effect of abnormalities in sympathetic, parasympathetic, chemoreceptor and renin-angiotensin function, and also reflects changes in respiratory pattern and physical inactivity [23]. Improvements in SDNN after exercise training may signal the normalization of these processes; small improvement in multiple mechanisms can cause a significant increase in SDNN [21]. Since, SDNN measurement provides prognostic information about morbidity and mortality in heart failure patients [23], an increased SDNN index indicates a valuable effect of the exercise training. The mechanisms by which exercise training improves HRV are not well understood. Two potential mediators have been considered to play a role in improving cardiac vagal modulation: nitric oxide and angiotensin II. Nitric oxide is thought to increase cardiac vagal control and to inhibit sympathetic influence indirectly, while angiotensin II is known to inhibit cardiac vagal tone. Exercise training has been demonstrated to increase nitric oxide bioavailability and to suppress angiotensin II [24-26] 
The ejection fraction correlated significantly only with one of the time domain parameters (SDNN). Similarly, a weak correlation between SDNN and the ejection fraction in patients after myocardial infarction was reported by Kleiger et al [27]. All parameters of cardiac function mirror the balance between the sympathetic and parasympathetic influences on the heart. There is an increase of sympathetic activity and a decrease in the vagal tone in heart failure leading to a reduction in SDNN, which appears early in cardiac dysfunction. This may explain the relationship between the ejection fraction, as a cardiac function parameter, and SDNN [28]. In the present study, the SDNN index is not strongly related to the ejection fraction. Modulation of the SDNN index by multiple factors including sympathetic, parasympathetic, neuroendocrine, and reninangiotensin functions may be the reasons of this weak relationship [23]. Measurement of SDNN in combination with other measurements might be a useful tool to assess the clinical condition of patients with heart failure. However, it remains unclear whether the improvement in SDNN is associated with the improvement in the ejection fraction in this group of patient. Our study was not designed to assess the ejection fraction, thus we were unable to examine the correlation between changes in SDNN and the ejection fraction.

The current study has several limitations. The first, we did not obtain follow-up data. The second, since this study included only patients with systolic heart failure, our results may not be applicable to individuals with diastolic heart failure. We suggest additional researches with larger group sizes and the follow-up assessment to determine whether the positive effects of exercise training are maintained with time.

\section{Conclusion}

The present study demonstrated that exercise training is safe and feasible in post percutaneous coronary intervention patients, even in those with the reduced ejection fraction. Heart failure patients after percutaneous coronary intervention can achieve a significant improvement of heart rate variability from a short-term exercise training program (seven weeks). Furthermore, one of the sensitive HRV indices, SDNN, and left ventricular ejection fraction are correlated weakly in these patients.

\section{Acknowledgment}

We thank the personnel at the Cardiac Rehabilitation Department of Al-Zahra Heart Charity Hospital for their cooperation. This article is based on the $\mathrm{PhD}$ thesis of Sara AbolahrariShirazi (proposal No.94-7578).

\section{Conflict of Interest}

\section{None}

\section{References}

1. Kolh P, Windecker S, Alfonso F, Collet JP, Cremer J, Falk V, et al. 2014 ESC/EACTS Guidelines on myocardial revascularization: the Task Force on Myocardial Revascularization of the European Society of Cardiology (ESC) and the European Association for Cardio-Thoracic Surgery (EACTS). Developed with the special contribution of the European Association of Percutaneous Cardiovascular Interventions (EAPCI). Eur J Cardiothorac Surg. 2014;46:517-92. doi: 10.1093/ejcts/ezu366. PubMed PMID: 25173601.

2. He XM, Chen L, Luo JB, Feng XX, Zhang YB, Chen $Q J$, et al. Effects of rhBNP after PCI on non-invasive hemodynamic in acute myocardial infarction patients with left heart failure. Asian Pac J Trop Med. 2016;9:791-5. doi: 10.1016/j.apjtm.2016.06.006. PubMed PMID: 27569890.

3. Kelly DJ, Gershlick T, Witzenbichler B, Guagliumi G, Fahy $M$, Dangas $G$, et al. Incidence and predictors of heart failure following percutaneous coronary intervention in ST-segment elevation myocardial infarction: the HORIZONS-AMI trial. Am Heart J. 2011;162:663-70. doi: 10.1016/j.ahj.2011.08.002. PubMed PMID: 21982658.

4. Jewiss D, Ostman C, Smart NA. The effect of resistance training on clinical outcomes in heart failure: A systematic review and meta-analysis. Int J Cardiol. 2016;221:674-81. doi: 10.1016/j.ijcard.2016.07.046. PubMed PMID: 27423089.

5. Smart N, Marwick TH. Exercise training for patients with heart failure: a systematic review of 
factors that improve mortality and morbidity. $A m$ $J$ Med. 2004;116:693-706. doi: 10.1016/j.amjmed.2003.11.033. PubMed PMID: 15121496.

6. Lloyd-Williams F, Mair FS, Leitner M. Exercise training and heart failure: a systematic review of current evidence. Br J Gen Pract. 2002;52:47-55. PubMed PMID: 11791816; PubMed Central PMCID: PMC1314201.

7. van Tol BA, Huijsmans RJ, Kroon DW, Schothorst $M$, Kwakkel $G$. Effects of exercise training on cardiac performance, exercise capacity and quality of life in patients with heart failure: a meta-analysis. Eur J Heart Fail. 2006;8:841-50. doi: 10.1016/j.ejheart.2006.02.013. PubMed PMID: 16713337.

8. Haykowsky MJ, Liang Y, Pechter D, Jones LW, McAlister FA, Clark AM. A meta-analysis of the effect of exercise training on left ventricular remodeling in heart failure patients: the benefit depends on the type of training performed. J Am Coll Cardiol. 2007;49:2329-36. doi: 10.1016/j.jacc.2007.02.055. PubMed PMID: 17572248.

9. Hambrecht R, Hilbrich L, Erbs S, Gielen S, Fiehn $\mathrm{E}$, Schoene N, et al. Correction of endothelial dysfunction in chronic heart failure: additional effects of exercise training and oral L-arginine supplementation. J Am Coll Cardiol. 2000;35:706-13. PubMed PMID: 10716474.

10. Piepoli MF, Davos C, Francis DP, Coats AJ, ExTra MC. Exercise training meta-analysis of trials in patients with chronic heart failure (ExTraMATCH). BMJ. 2004;328:189. doi: 10.1136/ bmj.37938.645220.EE. PubMed PMID: 14729656; PubMed Central PMCID: PMC318480.

11. Forslund L, Bjorkander I, Ericson M, Held C, Kahan T, Rehnqvist N, et al. Prognostic implications of autonomic function assessed by analyses of catecholamines and heart rate variability in stable angina pectoris. Heart. 2002;87:415-22. PubMed PMID: 11997407; PubMed Central PMCID: PMC1767117.

12. Mäkikallio TH, Huikuri HV, Hintze U, Videbæk J, Mitrani RD, Castellanos A, et al. Fractal analysis and time-and frequency-domain measures of heart rate variability as predictors of mortality in patients with heart failure. Am J Cardiol. 2001;87:178-82.

13. Weber F, Schneider H, von Arnim T, Urbaszek W. Heart rate variability and ischaemia in patients with coronary heart disease and stable angina pectoris; influence of drug therapy and prognostic value. TIBBS Investigators Group. Total Ischemic Burden Bisoprolol Study. Eur Heart J. 1999;20:38-50. PubMed PMID: 10075140.

14. Munk PS, Butt N, Larsen Al. High-intensity interval exercise training improves heart rate variability in patients following percutaneous coronary intervention for angina pectoris. Int J Cardiol. 2010;145:3124. doi: 10.1016/j.ijcard.2009.11.015. PubMed PMID: 19962772.

15. Tsai MW, Chie WC, Kuo TB, Chen MF, Liu JP, Chen $\mathrm{TT}$, et al. Effects of exercise training on heart rate variability after coronary angioplasty. Phys Ther. 2006;86:626-35. PubMed PMID: 16649887.

16. Beckers PJ, Denollet J, Possemiers NM, Wuyts FL, Vrints CJ, Conraads VM. Combined endurance-resistance training vs. endurance training in patients with chronic heart failure: a prospective randomized study. Eur Heart J. 2008;29:1858-66. doi: 10.1093/eurheartj/ehn222. PubMed PMID: 18515805.

17. Feiereisen $P$, Delagardelle $C$, Vaillant $M$, Lasar $Y$, Beissel J. Is strength training the more efficient training modality in chronic heart failure? Med Sci Sports Exerc. 2007;39:1910-7. doi: 10.1249/mss.0b013e31814fb545. PubMed PMID: 17986897.

18. Piepoli MF, Conraads V, Corra U, Dickstein K, Francis DP, Jaarsma $T$, et al. Exercise training in heart failure: from theory to practice. A consensus document of the Heart Failure Association and the European Association for Cardiovascular Prevention and Rehabilitation. Eur J Heart Fail. 2011;13:34757. doi: 10.1093/eurjhf/hfr017. PubMed PMID: 21436360.

19. Selig SE, Levinger I, Williams AD, Smart N, Holland DJ, Maiorana $A$, et al. Exercise \& Sports Science Australia Position Statement on exercise training and chronic heart failure. $J S c i$ Med Sport. 2010;13:288-94. doi: 10.1016/j. jsams.2010.01.004. PubMed PMID: 20227917.

20. Adamopoulos S, Ponikowski P, Cerquetani E, Piepoli M, Rosano G, Sleight $P$, et al. Circadian pattern of heart rate variability in chronic heart failure patients. Effects of physical training. Eur Heart $\mathrm{J}$. 1995;16:1380-6. PubMed PMID: 8746907.

21. Larsen Al, Gjesdal K, Hall C, Aukrust P, Aarsland $T$, Dickstein K. Effect of exercise training in patients with heart failure: a pilot study on autonomic balance assessed by heart rate variability. Eur $J$ Cardiovasc Prev Rehabil. 2004;11:162-7. PubMed PMID: 15187821.

22. Murad K, Brubaker PH, Fitzgerald DM, Morgan TM, Goff DC, Jr., Soliman EZ, et al. Exercise training improves heart rate variability in older patients with heart failure: a randomized, controlled, singleblinded trial. Congest Heart Fail. 2012;18:192-7. doi: 10.1111/j.1751-7133.2011.00282.x. PubMed 
PMID: 22536936; PubMed Central PMCID: PMC3400715.

23. Nolan J, Batin PD, Andrews R, Lindsay SJ, Brooksby $P$, Mullen $M$, et al. Prospective study of heart rate variability and mortality in chronic heart failure: results of the United Kingdom heart failure evaluation and assessment of risk trial (UK-heart). Circulation. 1998;98:1510-6. PubMed PMID: 9769304.

24. Buch AN, Coote JH, Townend JN. Mortality, cardiac vagal control and physical training--what's the link? Exp Physiol. 2002;87:423-35. PubMed PMID: 12392106.

25. Kingwell BA. Nitric oxide as a metabolic regulator during exercise: effects of training in health and disease. Clin Exp Pharmacol Physiol. 2000;27:239-
50. PubMed PMID: 10779120.

26. Townend JN, al-Ani M, West JN, Littler WA, Coote $\mathrm{JH}$. Modulation of cardiac autonomic control in humans by angiotensin II. Hypertension. 1995;25:1270-5. PubMed PMID: 7768573.

27. Kleiger RE, Stein PK, Bigger JT, Jr. Heart rate variability: measurement and clinical utility. Ann Noninvasive Electrocardiol. 2005;10:88-101. doi: 10.1111/j.1542-474X.2005.10101.X. PubMed PMID: 15649244.

28. Enciu EC, Stanciu SM, Matei D, Costache A. Prognostic markers in the pathology of cardiac failure: echocardiography and autonomic nervous system dysfunction. Rom J Morphol Embryol. 2015;56:401-6. PubMed PMID: 26193205. 